\title{
Nonlinear multi-scale modelling, simulation and validation of 3D knitted textiles
}

\author{
Oliver Weeger ${ }^{1,+}$, Amir Hosein Sakhaei ${ }^{1,2}$, Ying Yi Tan ${ }^{1}$, Yu Han Quek ${ }^{1}$, \\ Tat Lin Lee ${ }^{1}$, Sai-Kit Yeung ${ }^{1}$, Sawako Kaijima ${ }^{1,3}$, Martin L. Dunn ${ }^{1,4}$ \\ ${ }^{1}$ Singapore University of Technology and Design, SUTD Digital Manufacturing and Design Centre, \\ 8 Somapah Road, Singapore 487372, Singapore \\ ${ }^{2}$ University of Exeter, College of Engineering, Mathematics and Physical Sciences, \\ Harrison Building, North Park Road, Exeter EX4 4QF, United Kingdom \\ ${ }^{3}$ Harvard University, Graduate School of Design, \\ 48 Quincy St, Cambridge, MA 02138, USA \\ ${ }^{4}$ University of Colorado Denver, College of Engineering and Applied Science \\ P.O. Box 173364, Campus Box 104, Denver, CO 80217-3364, USA
}

\begin{abstract}
Three-dimensionally (3D) knitted technical textiles are spreading into industrial applications, since their geometric, structural and functional performance can be tailored and optimized on fibre-, yarnand fabric levels by customizing yarn materials, knit patterns and geometric shapes. The ability to simulate their complex mechanical behaviour is thus an essential ingredient in the development of a digital workflow for optimal design and manufacture of 3D knitted textiles. Here, we present a multi-scale modelling and simulation framework for the prediction of the nonlinear orthotropic mechanical behaviour of single jersey knitted textiles and its experimental validation. On the meso-scale, representative volume elements (RVEs) of the fabric are modelled as single, interlocked yarn loops and their mechanical deformation behaviour is homogenized using periodic boundary conditions. Yarns are modelled as nonlinear 3D beam elements and numerically discretized using an isogeometric collocation method, where a frictional contact formulation is used to model inter-yarn interactions. On the macro-scale, fabrics are modelled as membrane elements with nonlinear orthotropic material behaviour, which is parameterized by a response surface constitutive model obtained from the meso-scale homogenization. The input parameters of the yarn-level simulation, i.e., mechanical properties of yarns and geometric dimensions of yarn loops in the fabrics, are determined experimentally and subsequent meso- and macro-scale simulation results are evaluated against reference results and mechanical tests of knitted fabric samples. Good agreement between computational predictions and experimental results is achieved for samples with varying stitch values, thus validating our novel computational approach combining efficient meso-scale simulation using 3D beam modelling of yarns with numerical homogenization and nonlinear orthotropic response surface constitutive modelling on the macroscale.
\end{abstract}

Keywords: 3D knitting, technical textiles, digital design, multi-scale modelling, homogenization

\footnotetext{
+ Corresponding author. Tel.: +6581476749 .

E-mail address: oliver_weeger@sutd.edu.sg.
}

This version of the article has been accepted for publication, after peer review (when applicable) and is subject to Springer Nature's AM terms of use, but is not the Version of Record and does not reflect post-acceptance improvements, or any corrections. The Version of Record is available online at: https://doi.org/10.1007/ 


\section{Introduction}

Beyond the traditional textile industries such as apparel and fashion, there has been increasing interest in technical applications of textiles and textile composites in aerospace, architecture, biomedical, civil engineering, defence, marine and medical industries. These functional, technical and three-dimensional (3D) textiles provide a wide range of desirable properties for those industries and their applications, such as structural integrity, tailorable mechanical behaviour and designed anisotropy, as well as 3D shape-ability [1].

Among the techniques used for the fabrication of such technical textiles, i.e. braiding, knitting, weaving, and non-weaving, knitting has to-date a fairly modest market share [2]. However, with the latest advances in whole garment, computerized numerical control (CNC) knitting machine technology, it is now possible to knit complex 3D shapes [3] using high performance yarns made from materials such as carbon, glass, metals and shape memory alloys for advanced technical applications such as active fabrics [4], artificial muscles [5] or auxetic structures [6].

Adopting the flexibility of the $\mathrm{CNC}$ knitting technology with respect to yarn types and materials, knitting patterns and their parameters, as well as their seamless geometric shape-ability, will thus have great impact on the usage and application of these technical textiles. However, this will require advanced design, modelling, simulation, optimization and fabrication capabilities and computational tools that consider the complex mechanical behaviour of knitted textiles which arises from the interplay of geometry and material. An important aspect is the multi-scale nature of this behaviour. For knitted fabrics, three scales are typically distinguished: the composition of a yarn in terms of fibres and plies at the micro-scale (fibre-level), the intermeshing of yarns into a knitting pattern at the meso-scale (yarn-level), and the repetition and geometric variation of patterns forming a fabric on the macro-scale (fabric-level).

In this paper, we aim at an important step towards computational design and optimization for the fabrication of 3D knitted textiles. We focus on single jersey, plain weft-knitted textiles and establish a multiscale simulation framework for the prediction of their nonlinear orthotropic mechanical behaviour. Previous works characterizing the meso-scale mechanics and tensile properties of knitted textiles include analytical models where yarns are modelled using elastica theory [7], numerical methods where yarns are modelled using elastica [8], string structure [9] or beam models [10], as well as finite element methods where yarns are modelled as elastic continua [11]-[13]. These methods are then either used to model and simulate a whole fabric based on the meso-scale representation [8], [9], [13], or to homogenize its behaviour based on the simulation of representative volume elements (RVEs) [7], [10]-[12]. While the former approach is computationally expensive, the latter can only be extended to non-uniform tensile loading and complex shapes of textiles by using homogenized constitutive properties in a nonlinear multi-scale simulation framework [14]. Concurrent multi-scale simulation, where many meso-scale problems have to be solved in the course of solving a macro-scale problem, is again computationally very expensive. The sequential multiscale approach, however, requires a constitutive model that captures the nonlinear orthotropic behaviour of knitted textiles, which has to be based either on the homogenization of meso-scale RVE simulations or on phenomenological observations [15]. Here, we adopt the technique of response surface constitutive models 
[16], [17], where either the strain energy function or the stress-strain relationship are interpolated or approximated based on meso-scale RVE simulations.

By combining the meso-scale modelling of yarns as 3D beams with numerical homogenization of mesoscopic RVE behaviour using periodic boundary conditions and modelling of the macroscopic textile behaviour using a nonlinear orthotropic response surface material modelling, we introduce an efficient and yet accurate sequential multi-scale simulation framework for knitted textiles. The parameters of the mesoscopic model, e.g., Young's modulus and diameter of the yarn, stitch value or stitch type, can be easily modified to homogenize and characterize the mechanical behaviour of 3D knitted textiles with varying yarn types and knitting parameters.

The further outline of this paper is as follows: In Section 3, we introduce the methods used in this work, i.e. the geometrical model for parametric description of single jersey knits (2.1), the meso-scale, yarn-level modelling and simulation approach based on modelling of yarns as nonlinear 3D beams (2.2), the macroscale, fabric-level modelling approach using a nonlinear membrane model (2.3), the derivation of a nonlinear orthotropic response surface constitutive model, which is based on homogenization of meso-scale simulations (2.4), and details on the yarns, knitted samples and measurement techniques employed for physical validation (2.5). In Section 3, we then present and discuss some results of our computational framework and their validation against mechanical tests performed with textiles knitted with different loop lengths. Finally, we conclude the paper with a summary and outlook of future research in Section 4.

\section{Materials and methods}

\subsection{Geometric modelling of knit patterns and yarns}

Various geometrical models have already been proposed and established for modelling of single loops of weft-knitted single jersey fabrics. In this work, we implement the model proposed by Vassiliadis et al. in [18] which was used for mechanical simulations in [11].

This model assumes that the yarn has a uniform, circular cross-section and features the two-fold symmetry of an interlocked loop. Using the input parameters of wale width $w$, course height $c$ and yarn radius $r$, as well as a free parameter $t$ that is chosen to minimize the loop length $l$, a quarter of the yarn centreline curve of a loop is modelled as three continuous, analytically defined arc sections (see [18] for details). Subsequently, these three curves are interpolated using a single B-Spline curve. Here, we typically use degree $p=4$ and $n=12$ control points for the B-Spline interpolation and ensure that the first and second derivatives at the ends of these quarter curves are continuous when replicated to form the complete unit cell of an interlocked loop, see Figure 1a.

Since the knitting machine settings only allow to specify a desired average loop length $l$, which is actually an output parameter of the geometric model, we need to identify the necessary input parameters $c$ and $w$. Thus, we developed an image processing algorithm that computes the average values over dozens of courses and wales from a given photo of a single jersey knitted fabric sample, see Figure 1b. Furthermore, we also measure the actual diameters of yarns used and their tensional Young's moduli $E_{0}$. Since the cross-sections 


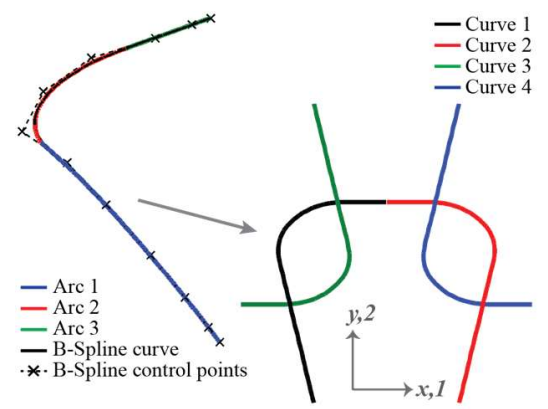

(a) Model for yarn centrelines

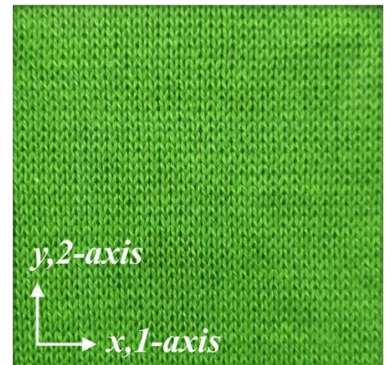

(b) $50 \times 50 \mathrm{~mm}$ photo

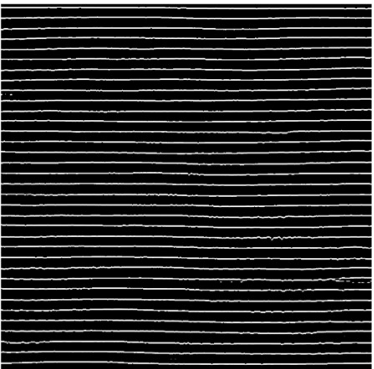

horizontal edges (courses)

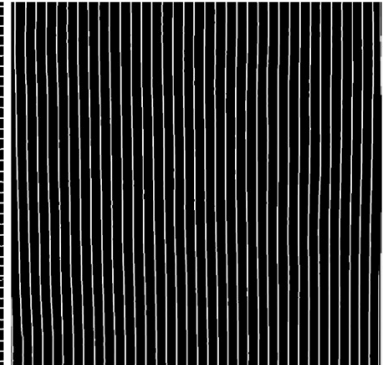

vertical edges (wales)

Figure 1: (a) Vassiliadis model for quarter loop is interpolated by a B-Spline curve (shown in 3D view) and then mirrored to generate a full loop model with 4 curves for the yarn centrelines (shown in plain $\mathrm{x} / \mathrm{y}$-view). (b) Image-based parameter identification of course height and wale width using edge detection.

of yarns are typically not perfectly round and have a rather elliptical shape, we consider their minimal radius $r_{0}$. However, in a tight knit, the yarn cross-sections are compressed at contact points and the geometrical model is only reasonable if $r \leq w / 8$. Thus, we choose $r=\min \left\{r_{0}, w / 8\right\}$.

\subsection{Meso-scale, yarn-level modelling and simulation}

In the meso-scale modelling step, the mechanical behaviour of representative volume element or unit cell of a knitted fabric, in this case a single interlocked loop, is simulated. Here, we use an approach based on the above-introduced geometrical model and the modelling of yarns using 3-dimensional beam theory.

As a compromise between the computationally expensive continuum modelling of yarns and the less accurate and versatile modelling using simple 1- or 2-dimensional beam theories, we employ the geometrically exact, elastic Simo-Reissner beam or Cosserat rod theory [19], which accounts for tension and shear deformation and is thus suitable for modelling both thick and thin 3D beams. It is based on representation of the rod as a framed curve described by its centreline, a spatial curve $\boldsymbol{r}(s):[0, L] \rightarrow \mathbb{R}^{3}$, and a frame $\boldsymbol{R}(s):[0, L] \rightarrow S O(3), \boldsymbol{R}=\left(\boldsymbol{d}_{1}, \boldsymbol{d}_{2}, \boldsymbol{d}_{3}\right)$ describing the orientation of the cross-sections along the centreline, see Figure 2a. Based on these kinematic variables in the current and initial configurations, given by $\boldsymbol{r}, \boldsymbol{R}$ and $\boldsymbol{r}_{0}, \boldsymbol{R}_{0}$ respectively, the translational (shear and tension) strains $\boldsymbol{\varepsilon}=\boldsymbol{R}^{\top} \boldsymbol{r}^{\prime}-\boldsymbol{R}_{0}^{\top} \boldsymbol{r}_{0}^{\prime}$ and rotational (curvature and twist) strains $\boldsymbol{\kappa}=\left\lfloor{\boldsymbol{R}^{\prime}}^{\top} \boldsymbol{R}\right\rfloor_{\times}-\left\lfloor\boldsymbol{R}_{0}^{\prime \top} \boldsymbol{R}_{0}\right\rfloor_{\times}$are calculated. Then, the corresponding stresses $\boldsymbol{\sigma}=\boldsymbol{A} \boldsymbol{\varepsilon}$ and $\chi=\boldsymbol{C} \boldsymbol{\kappa}$ are determined through linear elastic constitutive relationships and the governing equilibrium equations of linear and angular momentum are formulated in terms of the internal forces $\boldsymbol{n}=\boldsymbol{R} \boldsymbol{\sigma}$ and moments $\boldsymbol{m}=\boldsymbol{R} \boldsymbol{\chi}$ :

$$
\begin{gathered}
\boldsymbol{n}^{\prime}+\overline{\boldsymbol{n}}=\mathbf{0}, \\
\boldsymbol{m}^{\prime}+\boldsymbol{r}^{\prime} \times \boldsymbol{n}+\overline{\boldsymbol{m}}=\mathbf{0} . \quad \forall s \in(0, L),
\end{gathered}
$$

The geometric and material properties of the rod cross-sections, i.e. area $A=\pi r^{2}$, second moment of area $I=\pi r^{4} / 12$, Young's modulus $E$, and Poisson's ratio $v$, enter the formulation through the constitutive matrices $\boldsymbol{A}$ and $\boldsymbol{C}$. The elastic energy of the rod is given by $U=\frac{1}{2} \int_{0}^{L} \boldsymbol{\varepsilon}^{\top} \boldsymbol{\sigma}+\boldsymbol{\kappa}^{\top} \boldsymbol{\chi} d s$ and it shall be noted that in this way the initial configuration of the loop given by $\boldsymbol{r}_{0}, \boldsymbol{R}_{0}$ is assumed to be the relaxed configu- 


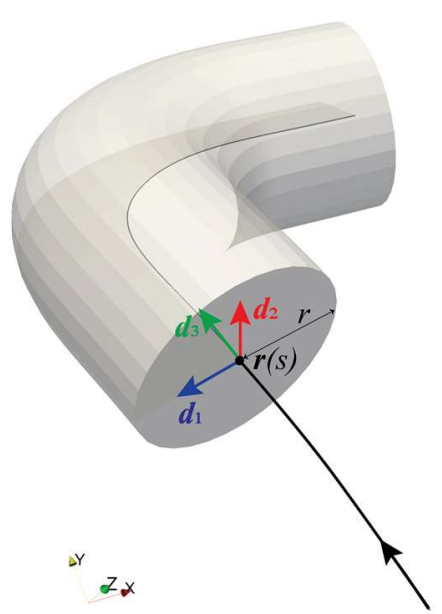

(a) Cosserat rod model of yarn

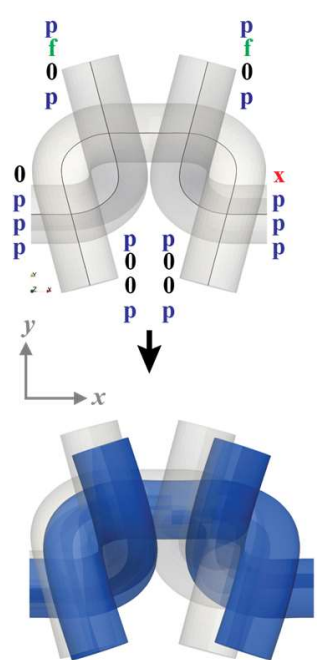

(b) course-wise

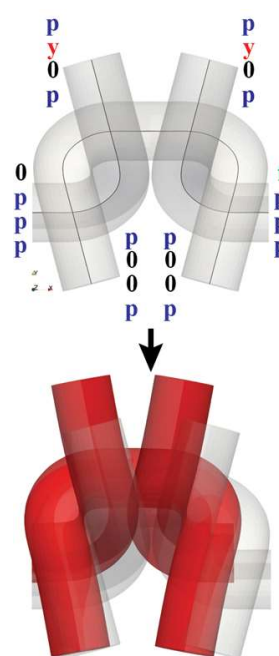

(c) wale-wise

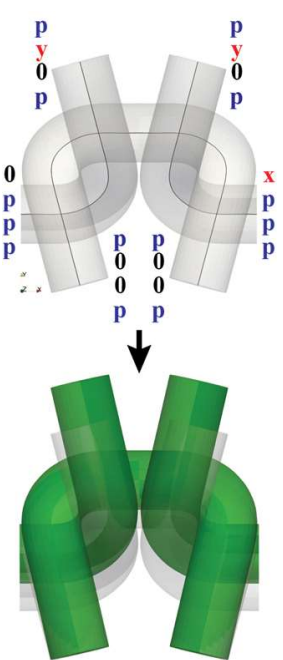

(d) biaxial

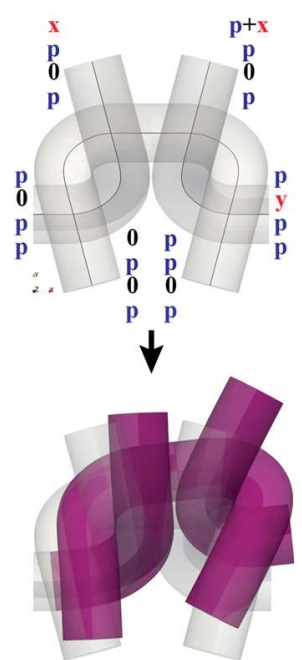

(e) pure shear

Figure 2: (a) Modelling of quarter loop yarn curve as Cosserat rod. (b-e) Boundary conditions and illustrations for course-wise and wale-wise uniaxial tension, biaxial tension, and pure shear. Each four characters next to a rod end indicate the applied boundary conditions for $\mathrm{x}-, \mathrm{y}-$, and $\mathrm{z}$-displacements and rotations (top to bottom, $0=$ zero-displacement, $\mathrm{p}=$ periodic, $\mathrm{f}=$ free, $\mathrm{x}=$-prescribed $\mathrm{x}$-displacement, $\mathrm{y}=$ prescribed $\mathrm{y}$-displacement).

ration of the yarn. Besides the balance equations, boundary conditions for $\boldsymbol{r}$ and/or $\boldsymbol{R}, \boldsymbol{m}, \boldsymbol{n}$ have to be applied. We apply periodic boundary conditions based on the type of analysis that is carried out, namely uniaxial tension in wale-wise (longitudinal) and course-wise (transversal) directions, biaxial tension, or pure shear, see Figure $2 b-e$.

For the numerical discretization and computational simulation of the governing equations of the Cosserat rod model we use an isogeometric collocation method [20], which is based on the representation of the kinematic variables using B-Spline and NURBS curves. This enables an efficient integration with the geometrical modelling procedure, as the B-Spline curve $\boldsymbol{r}_{0}$ obtained from the geometric model can be directly used in the computational method. Yarn-to-yarn contacts with frictional contact are also resolved within this framework, here with a penalty approach [21].

Important issues that affect the accuracy of the modelling approach using a 3D beam formulation are (a) the anisotropy of the yarns due their fibrous microstructure, (b) the change of cross-section radius from $r$ to $r_{0}$ due to compression and pre-strain of the yarns and (c) the rigidity of the cross-sections due the chosen mechanical model:

a) Similar to the approach taken in [11], we use two different values for the Young's modulus in the translational and rotational constitutive matrices $\boldsymbol{A}$ and $\boldsymbol{C}$, where $E_{A}=E_{0}$ is equal to the Young's modulus measured in the yarn tension tests and $E_{C}=0.5 E_{0}$. This approach is equivalent to assuming that the Young's modulus is graded within the cross-section, i.e. stiffer than $E_{0}$ in its centre and softer than $E_{0}$ at its boundary. This correlates to the actual fuzziness of fibrous and multi-ply yarns.

b) For consistent geometrical modelling of the loops, the yarn radius is described as $r=\min \left\{r_{0}, w / 8\right\}$. However, this potential decrease of the radius affects the translational and rotational stiffness of the 
mechanical model and thus we "balance" it by scaling the constitutive matrices by $\boldsymbol{A} \sim\left(r_{0} / r\right)^{2}$ and $\boldsymbol{C} \sim\left(r_{0} / r\right)^{4}$. Since the compression of the yarns originates from pre-tensioning during the knitting process, we scale the constitutive matrices again by $\boldsymbol{A}, \boldsymbol{C} \sim\left(r_{0} / r\right)^{2}$, which accounts for a nonlinear increase in Young's modulus when the yarn's a subject to tension strains.

c) The yarns cannot compress further during the simulated deformation process of the loops due to the rigidity of the cross sections in conventional beam formulations. However, this does not align with significant cross-section deformations that can be observed at contact points between yarn segments in physical fabrics, especially for multi-ply yarns. While the parameters of a contact penalty approach are typically chosen such that only a minimal penetration of contact surfaces occurs, we chose them to mirror the actual compressive properties of the yarns and allow for a certain amount of penetration that compensates for cross-section deformations. The contact penalty force takes the following form: $F=$ $k \frac{r}{p}\left(\frac{g}{r}\right)^{p}$, where $g$ is the penetrated distance, $k=0.01 E_{0}\left(r_{0} / r\right)^{8}$ and $p=3-\left(r_{0} / r\right)^{3}$.

As mentioned above, choices of those parameters are partially due to geometrical and mechanical considerations, and partially to best fit the experimental results obtained.

\subsection{Macro-scale, fabric-level modelling}

On the macro-scale, knitted fabrics show large deformation behaviour due to their general flexibility and complex constitutive behaviour which results from their microstructure. Thus, they can be modelled using thin shell or membrane models with nonlinear kinematics, large strains and hyperelastic constitutive laws.

Since fabrics are in general considered thin, the Kirchhoff hypothesis applies and the shell continuum can be modelled using Kirchhoff-Love theory by describing its midsurface $r: \mathcal{A} \subset \mathbb{R}^{2} \rightarrow \mathbb{R}^{3}$ and the normal vector field $\boldsymbol{n}: \mathcal{A} \rightarrow \mathbb{R}^{3}$, which always remains perpendicular to the mid-surface and is thus given in terms of the first and second derivatives of the surface, i.e. $\boldsymbol{n}\left(\xi_{1}, \xi_{2}\right)=\frac{\boldsymbol{r}_{1,} \times \boldsymbol{r}_{, 2}}{\left\|\boldsymbol{r}_{, 1} \times \boldsymbol{r}_{, 2}\right\|}$. Subsequently, the position of a point in the shell continuum is given as $\boldsymbol{x}: \mathcal{A} \times\left[-\frac{h}{2}, \frac{h}{2}\right] \rightarrow \mathbb{R}^{3}, \boldsymbol{x}\left(\xi_{1}, \xi_{2}, \xi_{3}\right)=\boldsymbol{r}\left(\xi_{1}, \xi_{2}\right)+$ $\xi_{3} \boldsymbol{n}\left(\xi_{1}, \xi_{2}\right)$, see Figure 3a, and the usual strain measures such as deformation gradient $\boldsymbol{F}=\frac{d \boldsymbol{x}}{d \boldsymbol{x}_{0}}$, CauchyGreen strain tensor $\boldsymbol{C}=\boldsymbol{F}^{\top} \boldsymbol{F}$ and Green-Lagrange (GL) strain tensor $\boldsymbol{E}=\frac{1}{2}(\boldsymbol{C}-\boldsymbol{I})$ can be computed. These are used to evaluate hyperelastic constitutive laws, which are typically defined using a strain-energy function $\psi$, and consequently the second Piola-Kirchhoff (PK2) stress tensor is given as $\boldsymbol{S}=\frac{d \psi}{d \boldsymbol{E}}=2 \frac{d \psi}{d \boldsymbol{C}}$. Further details on the subsequent formulation of balance equation and finite element discretization using an isogeometric approach can be found in [22].

\subsection{Orthotropic response surface material model and homogenization}

Simulating knitted textiles that are subjected to large strains and stresses requires nonlinear hyperelastic material modelling. This is complicated by their anisotropic behaviour due to the nature of the knitting process and its resulting microstructure that can cause substantially different material behaviour in course and wale directions. Modelling this nonlinear orthotropic constitutive behaviour is non-trivial, since most 


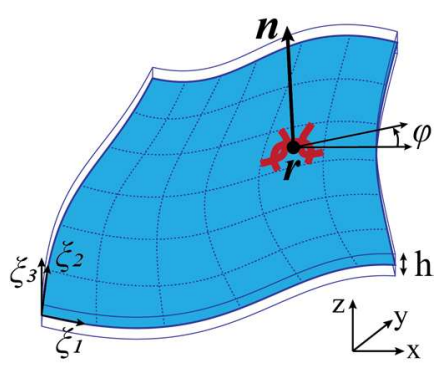

(a) Shell model

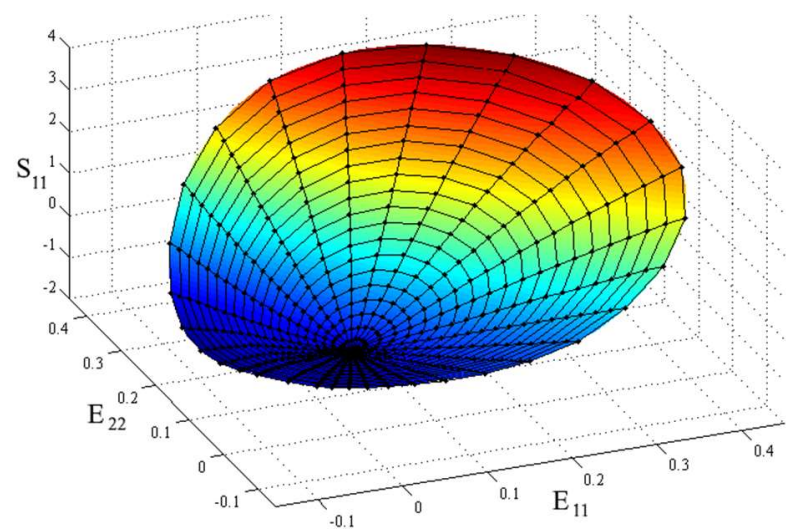

(b) Response surface $S_{11}\left(E_{11}, E_{22}\right)$

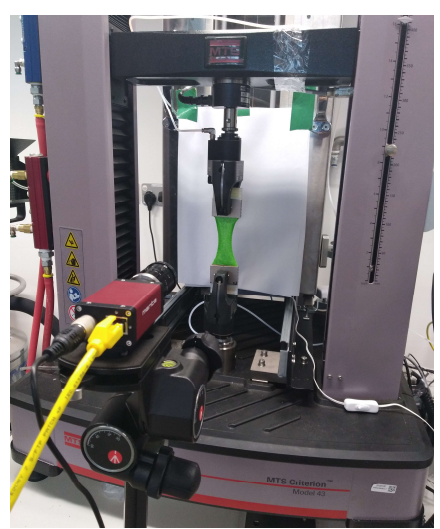

(c) Setup of fabric tensile test

Figure 3: (a) Shell model is described by midsurface $\boldsymbol{r}\left(\xi_{1}, \xi_{2}\right)$ (blue) and normal vector $\boldsymbol{n}\left(\xi_{1}, \xi_{2}\right)$. Orthotropic material direction $\varphi$ arising from meso-structure is illustrated by yarn loop (red). (b) Illustration of B-Spline response surface (colored) that interpolates data generated from biaxial simulations (wireframe). (c) Setup for fabric tests on MTS tensile testing machine with video extensometer.

commonly used hyperelastic material models are only applicable to isotropic materials, while general orthotropic material models are mostly only derived for linear constitutive behaviour.

In [15], an orthotropic strain energy function with 9 coefficients was presented for membrane models and fitted to textile measurement data, while in [16] the orthotropic strain energy function was parameterized using a response surface model that was also fitted to measurement data. We adopt a similar approach, where the second Piola-Kirchhoff (PK2) stress tensor is directly parameterized in terms of the Green-Lagrange (GL) strain tensor using a B-Spline response surface [17].

In this model, it is assumed that the shear components of the GL strain and PK2 stress are decoupled from the tensile strain and stress components when the textile is loaded along its orthotropic directions, where the 1-axis refers to the course- and the 2-axis to the wale-wise directions. Thus, the constitutive relationship between GL strain and PK2 stress tensors can be expressed in Voigt vector notation as:

$$
\boldsymbol{E}=\left(\begin{array}{c}
E_{11} \\
E_{22} \\
E_{12}
\end{array}\right), \quad \boldsymbol{S}=\left(\begin{array}{c}
S_{11} \\
S_{22} \\
S_{12}
\end{array}\right), \quad \boldsymbol{S}(\boldsymbol{E})=\left(\begin{array}{c}
S_{11}\left(E_{11}, E_{22}\right) \\
S_{22}\left(E_{11}, E_{22}\right) \\
S_{12}\left(E_{12}\right)
\end{array}\right)
$$

Consequently, we can parameterize the constitutive relationship $\boldsymbol{S}(\boldsymbol{E})$ using two response surfaces, $S_{11}\left(E_{11}, E_{22}\right)$ and $S_{22}\left(E_{11}, E_{22}\right)$, and one response curve, $S_{12}\left(E_{12}\right)$. Here, the response curves and surfaces are parameterized using tensor-product B-Splines, e.g.:

$$
S_{11}\left(E_{11}, E_{22}\right)=\sum_{i=1}^{n_{1}} \sum_{j=1}^{n_{2}} B_{i}\left(E_{11}\right) B_{j}\left(E_{22}\right) c_{i j}^{11},
$$

where $B_{i}$ 's are given B-Spline basis functions and $c_{i j}$ 's are the corresponding control points, see Figure $3 \mathrm{~b}$. Using splines of at least degree 2 and $C^{1}$-continuity ensures that the constitutive relationship is differentiable and the tangent stiffness matrix can be derived. 
To consider arbitrary, spatially varying orientations of the orthotropic material directions in our model, the GL strain $\widehat{\boldsymbol{E}}$ needs to be transformed from the global x,y-coordinate system to the local 1,2-coordinate system defining the orthotropic directions, $\boldsymbol{E}=\boldsymbol{T} \widehat{\boldsymbol{E}}$. Then, the local PK2 stress $\boldsymbol{S}(\boldsymbol{E})$ is evaluated using the response surfaces and finally transformed back to the global coordinate system, $\widehat{\boldsymbol{S}}=\boldsymbol{T}^{\top} \boldsymbol{S}$. Here, $\boldsymbol{T}$ is the transformation matrix that performs the rotation of the stress and strain tensors in Voigt notation by the angle $\phi$ between orthotropic directions with global directions, see Figure 3a. We have implemented this approach in Abaqus/CAE using a user-defined material subroutine (UMAT) for the CPS4R 4-node plane stress element.

To obtain the response surfaces and their points, we apply a homogenization approach. We carry out yarn-level simulations, where the macroscopic deformation gradients are prescribed to the RVEs and periodic boundary conditions are applied. The data for the $S_{11}$ and $S_{22}$ response surfaces can be obtained from biaxial tensile test simulations with prescribed, macroscopic deformation gradient $\boldsymbol{F}_{b a}$ and for $S_{12}$ it can be obtained from pure shear tests with deformation gradient $\boldsymbol{F}_{p s}$ :

$$
\boldsymbol{F}_{b a}=\left(\begin{array}{cc}
1+\varepsilon_{11} & 0 \\
0 & 1+\varepsilon_{22}
\end{array}\right), \quad \boldsymbol{F}_{p s}=\left(\begin{array}{cc}
1 & \varepsilon_{12} \\
\varepsilon_{12} & 1
\end{array}\right)
$$

where $\varepsilon_{11}$ and $\varepsilon_{22}$ are the prescribed tensile strains and $\varepsilon_{12}$ is the prescribed shear strain (see boundary conditions in see Figure $2 \mathrm{~d}+\mathrm{e}$ ). While the conversion from the prescribed strains $\varepsilon_{i j}$ and deformation gradients $\boldsymbol{F}_{b a}$ and $\boldsymbol{F}_{p s}$ to the GL strain $\boldsymbol{E}$ is straight forward,the resulting forces in both simulations and physical experiments are typically measured in the global coordinate system and the area in the undeformed configuration is used to obtain the first Piola-Kirchhoff (PK1) stress tensor $\boldsymbol{P}$, e.g., $P_{11}=F_{1} / A_{11}$, which then needs to be converted to the PK2 stress using the well-known formula $\boldsymbol{S}=\boldsymbol{F}^{-1} \boldsymbol{P}$.

\subsection{Knitting materials and testing methods}

For the physical validation of our multi-scale simulation framework, we fabricate single jersey, weftknitted fabric samples using a Shima Seiki MACH $2{ }^{\circledR} \mathrm{XS}$ computerized, 15-gauge whole garment knitting machine. Loop length per stitch is directly specified via the i-DSCS active yarn delivery control system. The yarn used across all samples is a 2-ply Ne 20/2's ring-spun cotton yarn, equivalent to a linear density of 59.1 Tex. We measure the loop length by unravelling and straightening several courses of the knitted fabrics to obtain the average loop length. A region of the fabric is also photographed to measure the course height and the wale width via image processing.

Subsequently, we prepare the fabric samples with minimized distortion and/or out-of-plane curling effects for tensile testing. The knitted fabrics are ironed to remove wrinkles and allowed to reach their dry relaxed state in an environment of $22{ }^{\circ} \mathrm{C}$ and $65 \%$ RH. 20 courses from the upper and lower regions of fabric are then removed and rectangular strips are cut out using a template from the unstretched pinned fabric. For this instance, we use $50 \times 50 \mathrm{~mm}$ samples to ensure that the fabric is not subjected to out-of-plane distortions which influences strain measurements. Moreover, additional sample lengths of 100, 150, $200 \mathrm{~mm}$ are also prepared for experimental testing. 


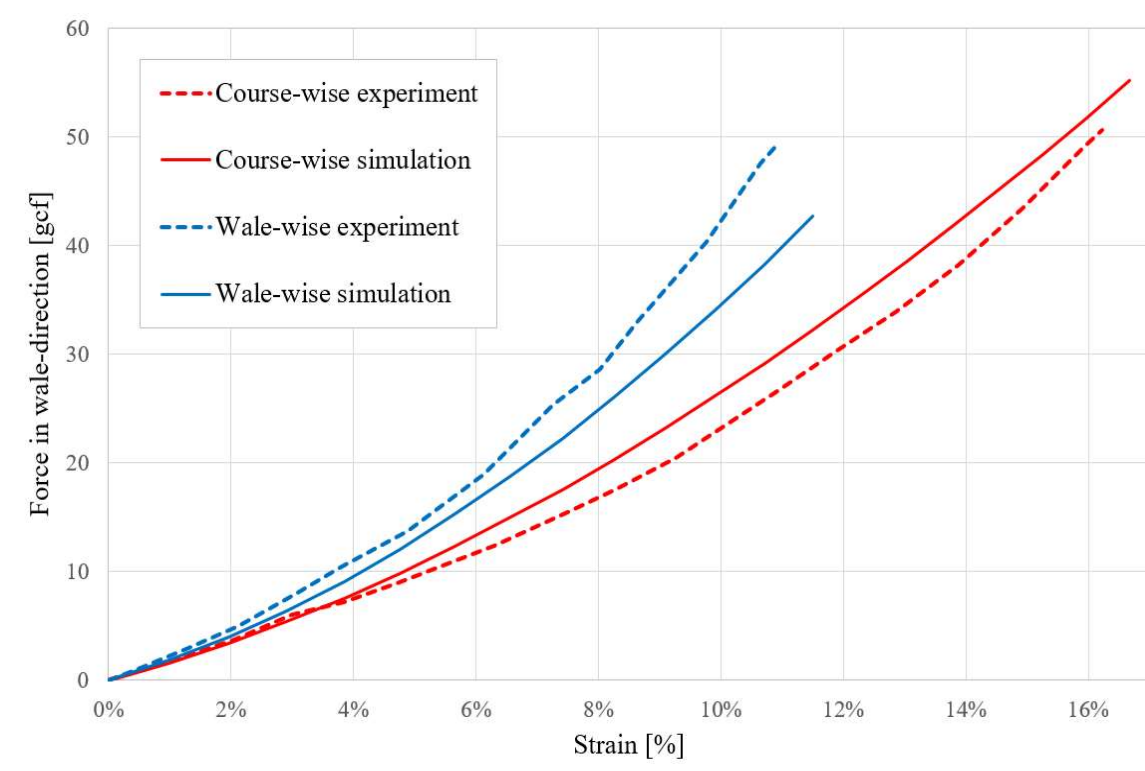

(a) Comparison of strain-force curves for reference experiments and simulations

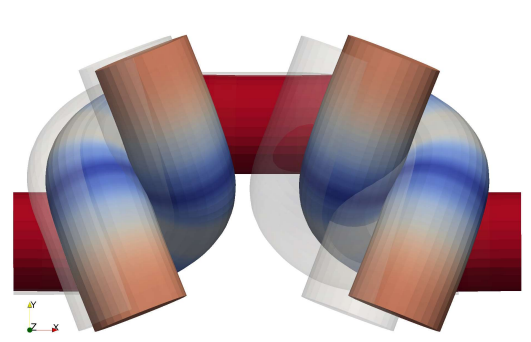

(b) Course-wise at $13.0 \%$ strain

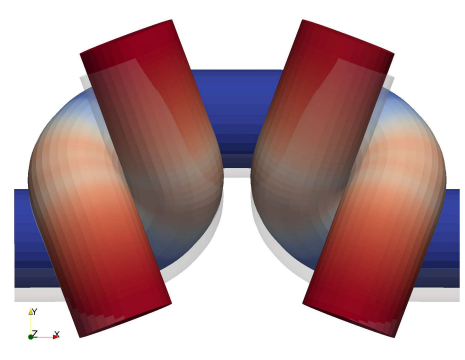

(c) Wale-wise at $9.1 \%$ strain

Figure 4: (a) Comparison of simulation results for course- and wale-wise strip biaxial tension tests against experimental reference results obtained in Vassiliadis et al. [11] and $(b+c)$ visualization of deformed RVE shapes.

We carry out uniaxial tensile tests on both the yarn and the fabric samples using the Instron 5943 and MTS Criterion universal testing machines, see Figure 3c. Samples are clamped in between pneumatic jaws and cross-head displacement is applied at a rate of $5.0 \mathrm{~mm} / \mathrm{min}$. To measure the strain, markers were attached near the centre of the sample and tracked using a calibrated video extensometer. These measurements showed good agreement with the strain being calculated from the crosshead movement. Since knitted fabrics are sensitive towards manufacturing uncertainties and variations, pre-tension and the curling effect we tested 3-5 samples for each loop length and obtained the reaction forces. Subsequently, we always plot the mean values of those measurements to validate the simulated results. Standard deviations were documented to be within the range of 5\%-20\%.

\section{Results and discussion}

For the application and validation of our multi-scale simulation framework for weft-knitted textiles, we consider two steps: first, we perform course- and wale-wise uniaxial tensile test simulations on the yarn-level and compare those results to reference results from literature and physical tests to assess the accuracy of the macro-scale simulation method and the homogenization approach. Secondly, we perform biaxial and pure shear simulations on the yarn-level RVEs to generate the response surface material models and subsequently use these for macro-scale simulations and validation.

\subsection{Yarn-level simulation and validations}

\section{Comparison against reference results}

To validate our meso-scale simulation approach, we first compare our method against reference results obtained from literature. We refer to the "sample 2" case in Vassiliadis et al. [11] with modelling parameters 


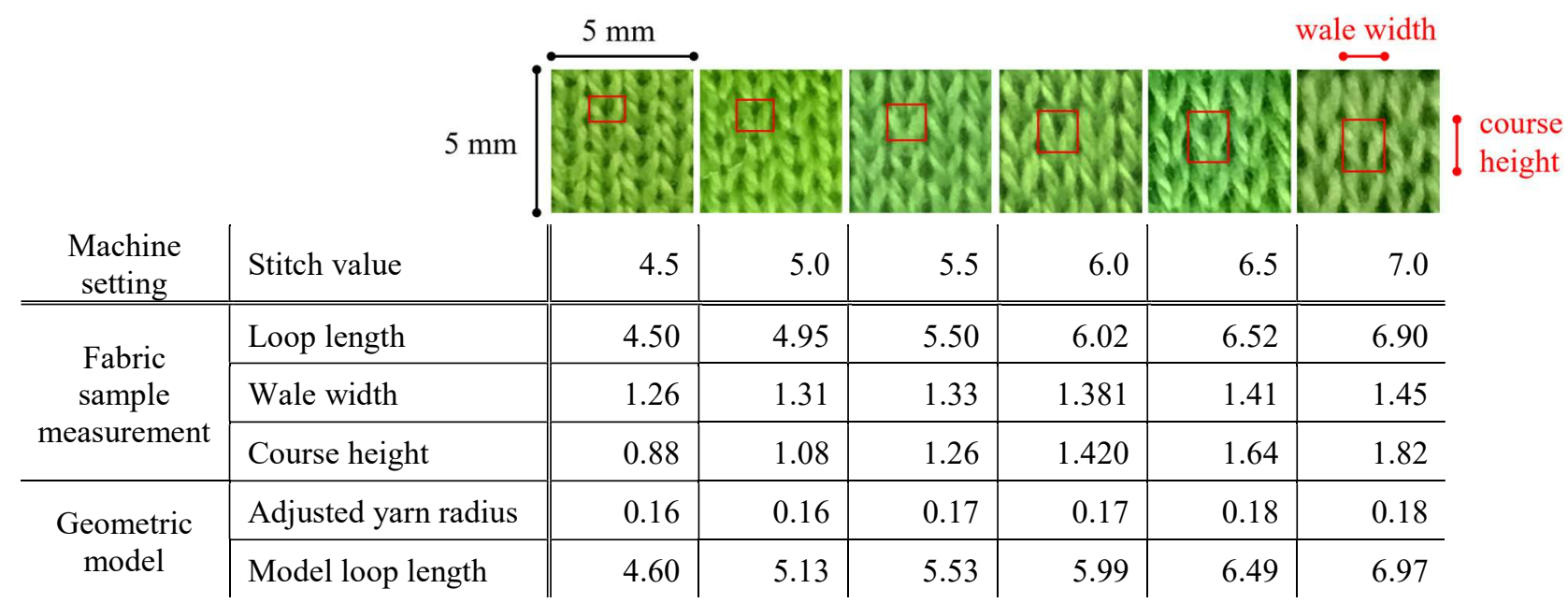

Table 1: Evaluation of geometric modelling parameters from fabric samples for varying stitch values from 4.5 to $7.0 \mathrm{~mm}$ (all values given in $\mathrm{mm}$ ). Pictures on top of table show $5 \times 5 \mathrm{~mm}$ images of the fabrics and boxes indicate size of a loop RVE.

$w=0.833 \mathrm{~mm}, c=0.486 \mathrm{~mm}, \ell=2.74 \mathrm{~mm}, r=r_{0}=0.0923 \mathrm{~mm}$ and Young's modulus $E=800 \mathrm{MPa}$. For the simulations, we use the isogeometric collocation method with a refined spline discretization with $p=$ $4, n=68$ and as contact friction parameter we use $\mu=0.15$. We perform strip-biaxial tension tests for both course- and wale-wise tension, i.e., along the 1-direction with strain ratios 1:0 and along the 2-direction with strain ratio $0: 1$, respectively, and plot the results in terms of strain-vs-force in $\mathrm{gf} / \mathrm{cm}$ in Figure 4 , along with the experimental results taken from [11]. Both strain-force curves show a good agreement with the experimental reference results with similar errors as the continuum finite element simulation results that are also provided in [11], see Figure 4a. This validates our meso-scale simulation method and its implementation.

\section{Simulations and tests for varying stitch values}

As a step towards modelling and optimizing technical fabrics with varying parameters, we now carry out meso-scale simulations for the fabric samples we have knitted with the cotton $20 \mathrm{~s} / 2$ yarns and varying stitch value machine settings ranging from 4.5 to $7.0 \mathrm{~mm}$.

First, we have determined the input parameters for the geometric model of the single jersey loop RVEs, namely wale width, course height and adjusted yarn radius, see Table 1. The measured loop lengths only deviate from the stitch value settings by a maximum of $1.4 \%$, and the resulting loop lengths of the generated B-Spline curves show slightly more deviation from the measured loop lengths at a maximum of $3.5 \%$. The adjusted yarn radius $r=\min \left\{r_{0}, w / 8\right\}$ is smaller than $r_{0}=0.19 \mathrm{~mm}$ in all cases, which means that all the fabrics are tight for the given stitch values.

Using the B-Spline curves generated for the centreline description of the yarns, we now perform uniaxial tensile test simulations for both course- and wale-directions, or 1- and 2-directions, respectively, for each stitch value for a strain range of $0-20 \%$. The B-Spline curves for the geometry have degree $p=4$ and $n=$ 12 , and are refined to $n=68$ for the isogeometric rod simulation. The material parameters are based on yarn tensile tests for the Young's modulus $E_{0}=80 \mathrm{MPa}$, Poisson's ratio is assumed as $v=0.3$, and the 


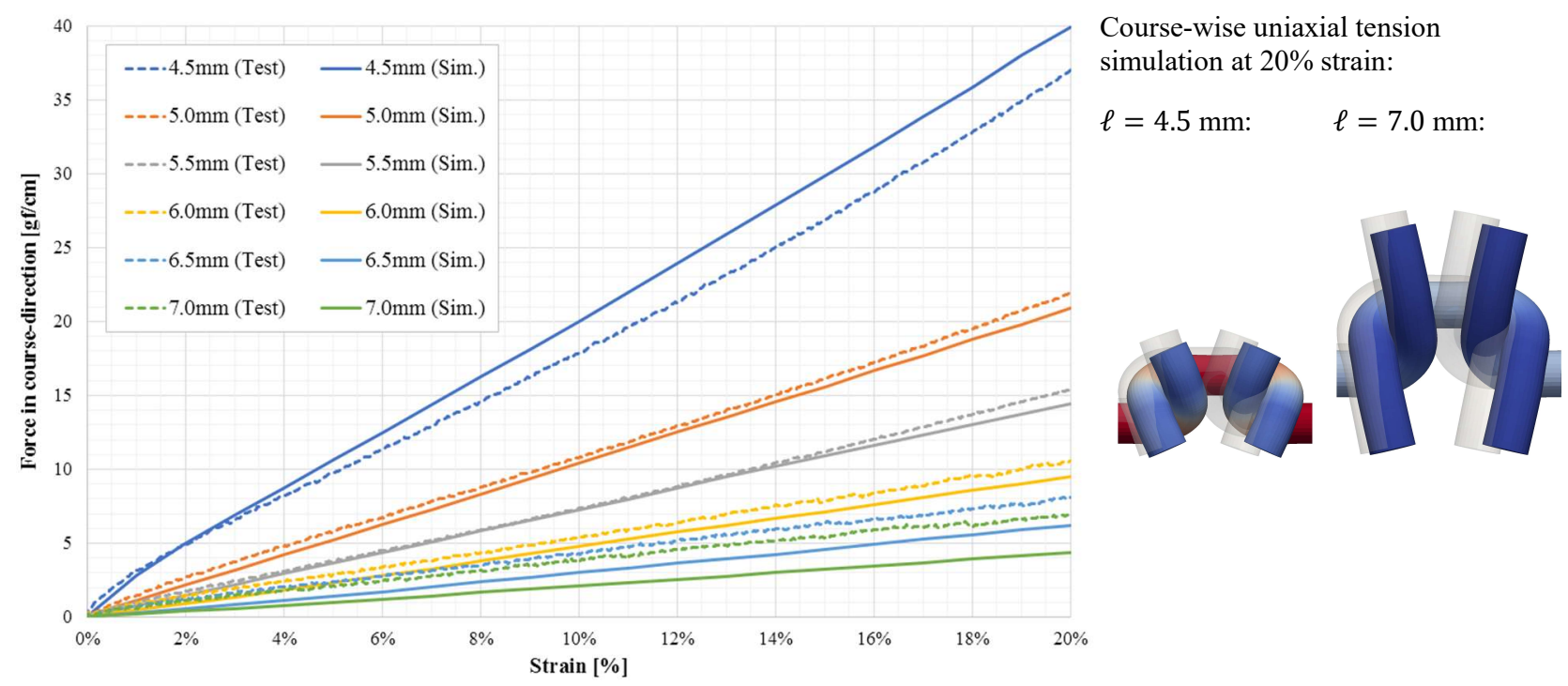

Figure 5: Comparison of experimental (dashed) and uniaxial simulation (solid) results for course-wise tension tests for varying loop length from 4.5 to $7.0 \mathrm{~mm}$. Example deformations at $20 \%$ strain shown for 4.5 $\mathrm{mm}$ and $7.0 \mathrm{~mm}$.

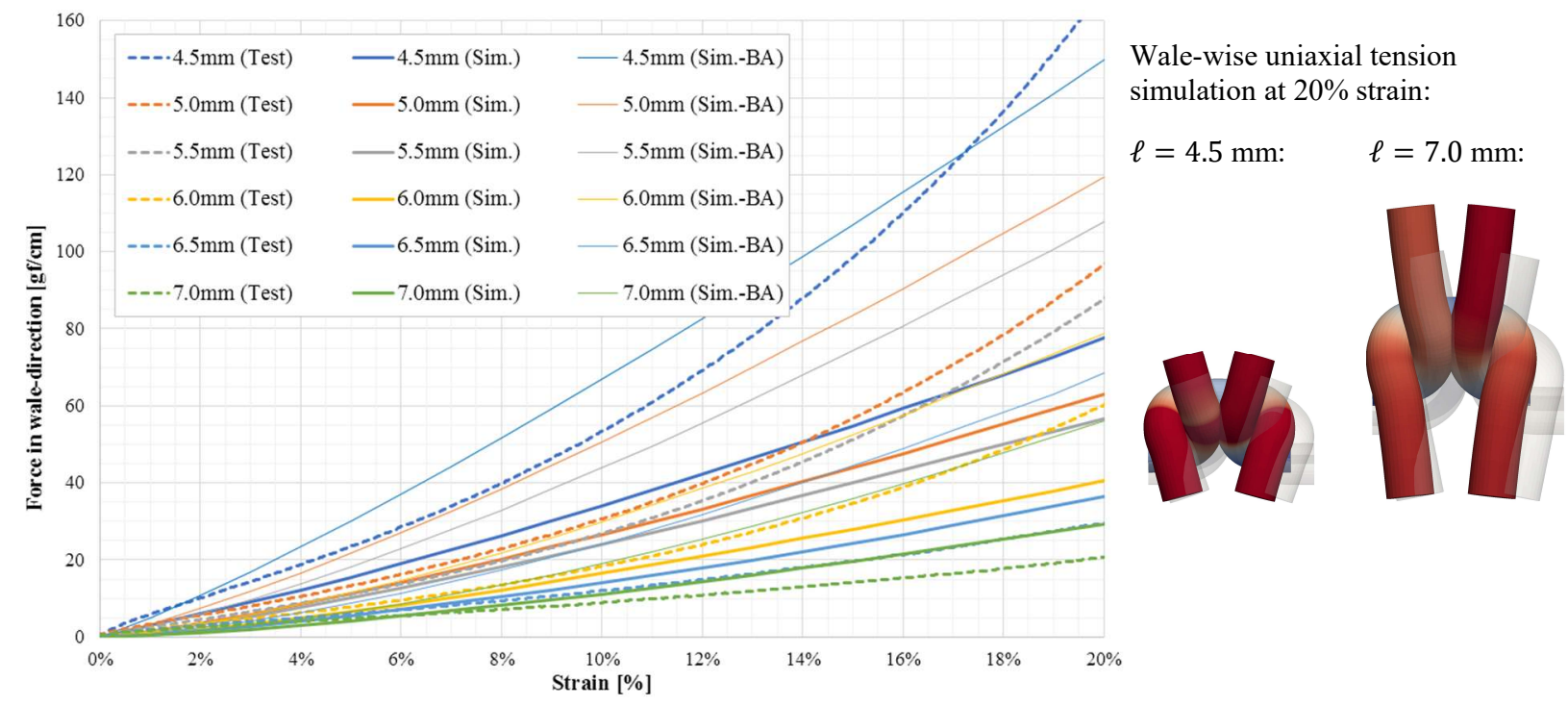

Figure 6: Comparison of experimental (dashed) and uniaxial (solid) and strip-biaxial (solid thin) simulation results for wale-wise tension tests for varying loop length from 4.5 to $7.0 \mathrm{~mm}$. Example deformations at $20 \%$ strain shown for $4.5 \mathrm{~mm}$ and $7.0 \mathrm{~mm}$.

parameters of the contact formulation are chosen as outlined above in Section 2.2 with friction parameter $\mu=0.15$. The runtime for one rod-based simulation with 21 incremental load steps to generate the strainforce curves is around 1-2 minutes on a standard, 8 core desktop PC, which is faster than a comparable, nonlinear continuum finite element simulation of around 15 minutes.

We compare the results of tensile tests performed with the physical knitted samples against simulations of uniaxial tensile tests, since our tested samples are much longer than the ones used in [11] and are thus closer to uniaxial rather than strip-biaxial strain state. The strain-force curves are reported in Figure 5 for course-wise and Figure 6 for wale-wise tension, where the strains values refer to the prescribed, macroscopic 
strains of the RVE in the simulation and to the engineering strain based on the cross-head deformation in the physical tensile tests. Force values are reported in $\mathrm{gf} / \mathrm{cm}$.

Overall, the comparison between experimental tests and computational simulations shows a good agreement and the trends of softening of the strain-force curves for increasing loop length are well captured. Especially for the course-wise tension tests, the qualitative and quantitative behaviour of the tests matches the uniaxial tension simulations well and both, the relatively linear behaviour of each strain-force curve and the trend for increasing loop length, are consistent in experiments and simulations. In the wale-wise tension case, the deviations between measurements and simulations tend to be larger and the trend for increasing loop length is less consistent. Although the nonlinear stiffening effect with increasing strain is quantitively well captured, the reaction forces of the tight fabric $(4.5 \mathrm{~mm})$ are underestimated, whereas for looser ones $(6.5 \mathrm{~mm}$ and $7.0 \mathrm{~mm})$ the forces are over-estimated. This could be related to the further tightening of the loops and compression of yarns occurring in the wale-wise tension scenario of tight knits, or nonlinear, strain-dependent behaviour of the Young's modulus or frictional coefficients of the yarns. Thus, we have also carried out strip-biaxial simulations in this case and plotted them in Figure 6, too. As expected, the biaxial simulations are stiffer than the uniaxial ones and tend to over-estimate the test results for looser fabrics, but match the experiments better for tighter knits with shorter loop length.

Furthermore, for stitch value $5.5 \mathrm{~mm}$, we have also determined the ratios of lateral versus transversal contractions at $20 \%$ strain from video-extensometer measurement made at the centre of the tested samples. For these "effective Poisson's ratios" $\bar{v}_{12}=-\frac{\varepsilon_{22}}{\varepsilon_{11}}$ in course-wise and $\bar{v}_{21}=-\frac{\varepsilon_{11}}{\varepsilon_{22}}$ in wale-wise tension, we obtain $\bar{v}_{12}=0.43$ and $\bar{v}_{21}=0.82$ in the simulations, which is in good agreement with the experimental observations of $\bar{v}_{12} \approx 0.41$ and $\bar{v}_{21} \approx 0.72$.

\subsection{Homogenization and fabric-level simulations}

Having validated the accuracy of the meso-scale simulations in the preceding section, we now move on to the generation of orthotropic response surface material models using homogenization on the yarn-level and to the application of those models in macro-scale simulations.

First, we begin with the generation of the data for the response surface material model. As discussed above, the response surfaces interpolate the stress-strain data obtained from biaxial and pure shear yarn-level RVE simulations. Here, we exemplify this process for the cotton $20 \mathrm{~s} / 2$ single jersey fabrics with stitch value $5.5 \mathrm{~mm}$. For fixed ratios of $\varepsilon_{11}: \varepsilon_{22}$, the biaxial tests are simulated by increasing the load factor from 0 to 1 . Thus, the data obtained is star-shaped in the $E_{11} / E_{22}$-domain and each simulation run is corresponding to a radial line from the centre to the boundary of the surface, see Figure 7. For shear tests, the shear strain $\varepsilon_{12}$ is increased from 0 to $30 \%$ to obtain the curves for $S_{12}\left(E_{12}\right)$. These data points are then interpolated by BSpline surfaces and curves, here of degree $p=2$, to obtain the response surface and curve parameterizations of the nonlinear orthotropic material model.

Finally, we use those material models in the macro-scale, textile level simulations. We simulate the tensile behaviour of a $50 \times 50 \mathrm{~mm}$ patch of the single jersey knitted cotton $20 \mathrm{~s} / 2$ yarn with stitch value 5.5 

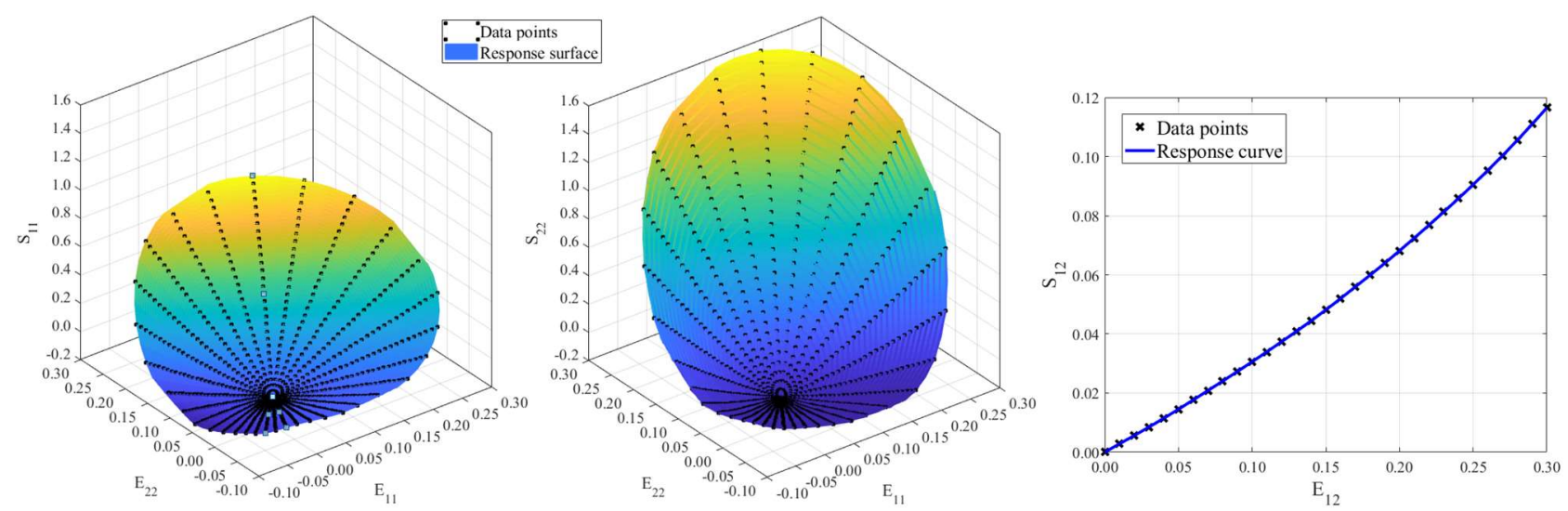

Figure 7: Homogenization of constitutive behaviour of single jersey knitted cotton $20 \mathrm{~s} / 2$ yarn at stitch value $5.5 \mathrm{~mm}$. Response surfaces $S_{11}\left(E_{11}, E_{22}\right)$ and $S_{22}\left(E_{11}, E_{22}\right)$ from biaxial simulations and response curve $S_{12}\left(E_{12}\right)$ from pure shear simulations are shown. Values of PK2 stress $\boldsymbol{S}$ are given in MPa*mm, GL strain $\boldsymbol{E}$ is dimensionless.

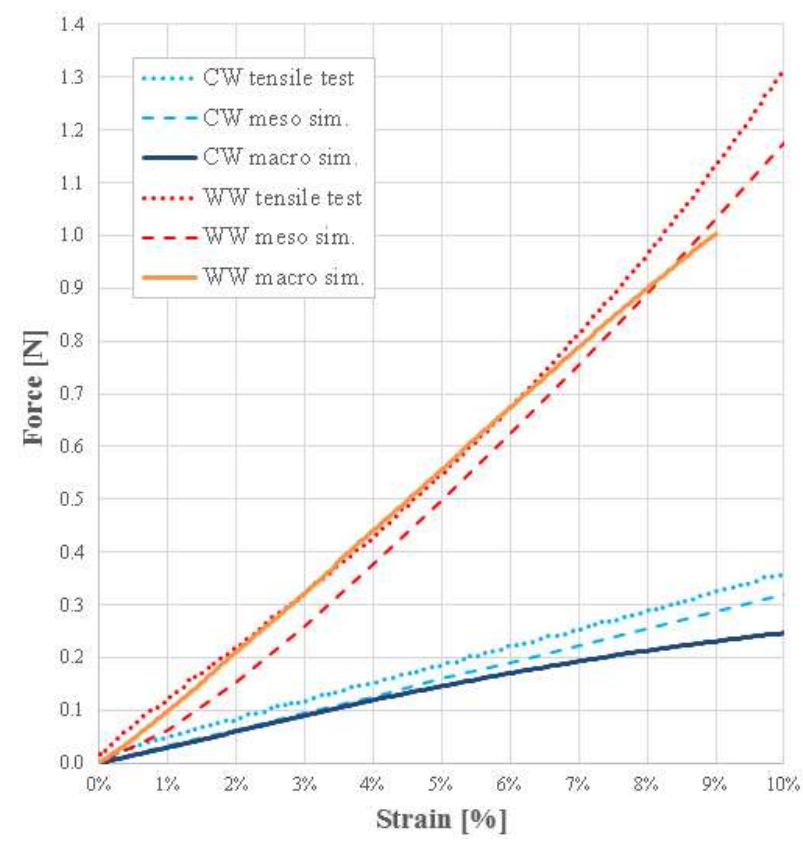

(a) Strain-force curves for course- and wale-wise tests
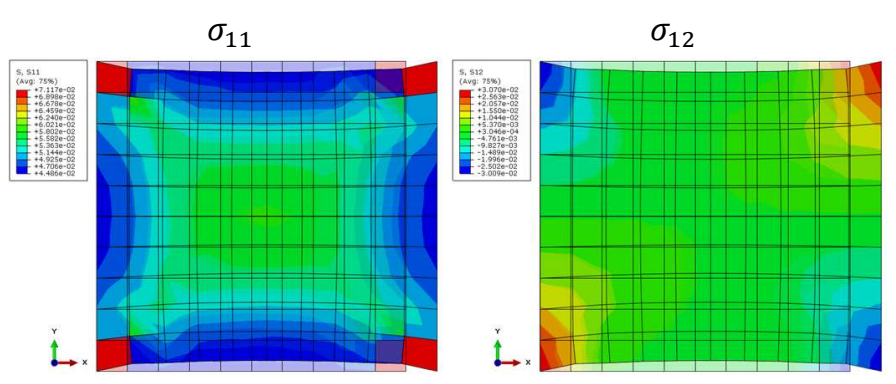

(b) Deformed shapes and stresses for course-wise tension
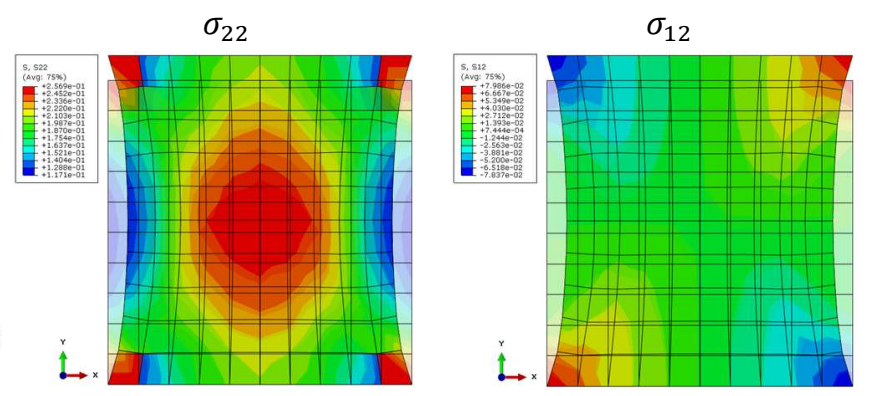

(c) Deformed shapes and stresses for wale-wise tension

Figure 8: Macro-scale simulation of 50x50 mm textile patch. (a) Comparison of strain-force curves of fabric-level simulations (macro sim.) with tensile tests and meso-scale simulation for both course- $(\mathrm{CW})$ and wale-wise (WW) tension. (b) Cauchy stress states $\sigma_{11}$ and $\sigma_{12}$ for course-wise at $10 \%$ nominal strain and (c) $\sigma_{22}$ and $\sigma_{12}$ for wale-wise tension at $9 \%$ strain.

$\mathrm{mm}$ along the course- and wale-wise directions. For course-wise, the patch is clamped at the left side and a uniform displacement is applied on the right boundary, while for wale-wise it is clamped at the bottom and a uniform displacement is applied on the top boundary. The results for using 10x10 finite elements are shown in Figure 8. The visualization of deformed shapes and Cauchy stresses at $10 \%$ and $9 \%$ applied, nominal strain for course- and wale-wise tension cases in Figure 8b-c indicate the coupling of tensile with shear modes for this type of applied boundary conditions. It can also be seen that the lateral contraction in the wale-wise tension case is much higher than in the course-wise case, which is in accordance with the earlier stated observations made regarding the effective Poisson's ratios. The comparison of the strain-force curves 
in Figure 8a shows good agreement of the macro-scale simulations with the actual tensile tests and the mesoscale, uniaxial tension simulations taken from Figure 5 and Figure 6. This serves as an initial validation of the multi-scale simulation approach.

\section{Conclusions}

In this work, we have presented a nonlinear multi-scale modelling and simulation framework for knitted textiles, which marks a first step towards the development of a digital workflow for design and fabrication of 3D knitted textiles. Our approach is based on the modelling and simulation of the meso-scale mechanical behaviour of knitted textiles using a nonlinear beam model to represent the yarns of a representative volume element of the stitch type, here being the single jersey knitting pattern. This approach was validated against reference results from existing literature and tensile tests with fabrics made from cotton yarn at various stitch values. Then, the yarn-level behaviour was homogenized to obtain a nonlinear, orhtotropic constitutive model for the macro-scale, which is represented through B-Spline response surface models. This material model was then employed in a nonlinear membrane formulation for fabric-scale simulations.

Next steps and future research directions include further validations of the multi-scale simulation approach for more complex loading scenarios and extending the macro-scale model and homogenization procedure to shell models including bending deformation, which will allow to simulate more complex 3dimensional fabric geometries and deformations. Furthermore, we want to generalize the approach towards geometric modelling of other stitch types on the meso-scale, e.g. double jersey, rib- and purl-based fabrics and inlaid knitting patterns. Ultimately, we aim at developing an optimization framework, that can vary the meso-scale parameters such as knitting pattern, stitch values and orientation to obtain a desired macroscopic behaviour of a knitted textile.

\section{Acknowledgement}

The authors acknowledge funding through the SUTD Digital Manufacturing and Design (DManD) Centre, supported by the Singapore National Research Foundation.

\section{References}

[1] K. H. Leong, S. Ramakrishna, Z. M. Huang, and G. A. Bibo, "The potential of knitting for engineering composites - a review," Composites Part A, vol. 31, no. 3, pp. 197-220, 2000.

[2] M. de Araujo, R. Fangueiro, and H. Hong, "Modelling and simulation of the mechanical behaviour of weft-knitted fabrics for technical applications," Autex Res. J., vol. 4, no. 2, 2004.

[3] J. Underwood, The Design of 3D Shape Knitted Performs. School of Fashion and Textiles, RMIT University, 2009.

[4] J. Abel, J. Luntz, and D. Brei, "Hierarchical architecture of active knits," Smart Mater. Struct., vol. 22, no. 12, 2013.

[5] E. J. Ball, M. A. Meller, J. B. Chipka, and E. Garcia, "Modeling and testing of a knitted-sleeve fluidic artificial muscle," Smart Mater. Struct., vol. 25, no. 11, 2016. 
[6] M. Glazzard and P. Breedon, "Weft-knitted auxetic textile design," Phys. Status Solidi B, vol. 251, no. 2, pp. 267-272, 2014.

[7] H. Hong, M. D. De Araujo, R. Fangueiro, and O. Ciobanu, "Theoretical analysis of load-extension properties of plain weft knits made from high performance yarns for composite reinforcement," Text. Res. J., vol. 72, no. 11, pp. 991-996, 2002.

[8] J. M. Kaldor, D. L. James, and S. Marschner, "Simulating knitted cloth at the yarn level," in $A C M$ Trans. Graphics (TOG), 2008, vol. 27, p. 65.

[9] V. Shiryaev and J. Orlik, "A one-dimensional computational model for hyperelastic string structures with Coulomb friction," Math. Meth. Appl. Sci., vol. 40, no. 3, pp. 741-756, 2017.

[10] J. Orlik, G. Panasenko, and V. Shiryaev, "Optimization of Textile-Like Materials via Homogenization and Beam Approximations," Multiscale Model. Simul., vol. 14, no. 2, pp. 637-667, 2016.

[11] S. G. Vassiliadis, A. E. Kallivretaki, and C. G. Provatidis, "Mechanical simulation of the plain weft knitted fabrics," Int. J. Clothing Sci. Technol., vol. 19, no. 2, pp. 109-130, Mar. 2007.

[12] S. Fillep, J. Orlik, Z. Bare, and P. Steinmann, "Homogenization in periodically heterogeneous elastic bodies with multiple micro-contact," Math. Mech. Solids, vol. 19, no. 8, pp. 1011-1021, 2014.

[13] D. Liu et al., "On the role of material architecture in the mechanical behavior of knitted textiles," Int. J. Solids Struct., vol. 109, pp. 101-111, 2017.

[14] M. G. D. Geers, V. G. Kouznetsova, K. Matouš, and J. Yvonnet, "Homogenization Methods and Multiscale Modeling: Nonlinear Problems," in Encyclopedia of Computational Mechanics, Second Edition., John Wiley \& Sons, Ltd., 2017.

[15] M. S. Yeoman, D. Reddy, H. C. Bowles, D. Bezuidenhout, P. Zilla, and T. Franz, "A constitutive model for the warp-weft coupled non-linear behavior of knitted biomedical textiles," Biomaterials, vol. 31, no. 32, pp. 8484-8493, Nov. 2010.

[16] A. M. Widhammer, Variation of Reference Strategy - Generation of Optimized Cutting Patterns for Textile Fabrics. Ingenieurfakultät Bau Geo Umwelt, Technische Universität München, 2015.

[17] M. Coelho, D. Roehl, and K.-U. Bletzinger, "Material model based on NURBS response surfaces," Appl. Math. Modell., vol. 51, pp. 574-586, Nov. 2017.

[18] S. Vassiliadis, A. E. Kallivretaki, and C. G. Provatidis, "Geometrical modelling of plain weft knitted fabrics," Indian J. Fibre \& Textile Res., vol. 32, pp. 62-71, 2007.

[19] S. Eugster, Geometric Continuum Mechanics and Induced Beam Theories, vol. 75. Springer International Publishing, 2015.

[20] O. Weeger, S.-K. Yeung, and M. L. Dunn, "Isogeometric collocation methods for Cosserat rods and rod structures," Comput. Methods Appl. Mech. Eng., vol. 316, pp. 100-122, 2017.

[21] O. Weeger, B. Narayanan, L. D. Lorenzis, J. Kiendl, and M. L. Dunn, “An isogeometric collocation method for frictionless contact of Cosserat rods," Comput. Methods Appl. Mech. Eng., vol. 321, pp. 361-382, 2017.

[22] J. Kiendl, M.-C. Hsu, M. C. H. Wu, and A. Reali, "Isogeometric Kirchhoff-Love shell formulations for general hyperelastic materials," Comput. Methods Appl. Mech. Eng., vol. 291, pp. 280-303, 2015. 